\title{
Disfonías espasmódicas: estudios acústicos
}

\author{
Liliana Sigal \\ Universidad de Buenos Aires, Argentina
}

\begin{abstract}
Resumen
La disfonía espasmódica es un desorden vocal severo caracterizado por una interrupción involuntaria de la fonación, denominada también distonía focal laríngea. En este estudio se presentan los resultados de una investigación sobre las características acústico-vocales de una población de pacientes con disfonías espasmódicas, y la comparación de las mismas con las de un grupo control (sujetos con el diagnóstico otorrinolaringológico de cuerdas vocales móviles, sin daños estructurales en la mucosa glótica, ni alteraciones neurológicas que involucren la dinámica cordal). Se analizó acústicamente la vocal /a/ investigándose: valor de la frecuencia fundamental, desviación estándar, rango diferencial entre frecuencia máxima y mínima, y número de quiebres fonatorios. Se utilizó para estas mediciones el Sistema de análisis acústico Praat (Boersma y Weenink, 2003). En los resultados no se encontraron diferencias significativas entre las medias de la Frecuencia Fundamental entre ambos grupos estudiados. La comparación de los promedios del desvío estándar de la Frecuencia Fundamental arrojó sin embargo diferencias significativas, así como la comparación de los promedios de los rangos entre frecuencia máxima y mínima. Respecto a la variable Quiebres Fonatorios, se calcularon solamente en el grupo de pacientes ya que ninguno de los sujetos del grupo control presentó interrupciones fonatorias en la emisión de la vocal. Cabe concluirse que la desviación estándar, el rango entre el valor máximo y mínimo de frecuencia fundamental y el número de interrupciones durante la emisión de vocal prolongada pueden utilizarse como indicadores de disfonía espasmódica.
\end{abstract}

Palabras clave: Disfonía espasmódica; Distonía laríngea; Medidas acústicas; Voz.

\section{Spasmodic dysphonia: Acoustic measures}

\begin{abstract}
Spasmodic dysphonia is a severe voice disorder characterized by involuntary disruption of phonation, also called focal laryngeal dystonia. This study presents the results of an investigation into the acousticvocal characteristics of a population of patients with spasmodic dysphonia, and comparing them with those of a control group (patients with diagnosis of mobile vocal cords, without damage in the structure of the glottal mucosa or neurological disorders involving the movement of the vocal cords). The following parameters were investigated in the emission of the vowel /a /: mean pitch value, standard deviation, minimum pitch, maximum pitch and a number of phonatory breaks. The Praat acoustic analysis system was used for these measurements (Boersma and Weenink, 2003). Regarding results, no significant differences were found between patients and control group for the average measures of mean pitch in both sexes. Comparison of the standard deviation of mean pitch and the ranges between maximum and minimum pitch revealed significant differences between the group of patients and controls. The voice breaks were calculated only in the group of patients. No person in the control group had phonatory disruptions in the emission of the vowel. The standard deviation of mean pitch, the range between maximum and minimum pitch and the number of phonatory breaks during prolonged vowel can be used as indicators of spasmodic dysphonia.
\end{abstract}

Key words: Acoustic measures; Laryngeal dystonia; Spasmodic dysphonia; Voice.

Correspondencia con la autora: lilianasigal@yahoo.com.ar

Recibido 29 Noviembre 2012. Primera revisión 2 Febrero 2013. Aceptado 23 Mayo 2013. 


\section{Introducción}

La disfonía espasmódica (DE en adelante) fue inicialmente denominada disfonía espástica por Traube, quien en 1871 caracterizó a esta disfonía como una ronquera de causa psicológica. Belhau y Pontes (2002) refieren que Aronson en el año 1968 propuso el nombre de Disfonía Espasmódica, argumentando que la espasticidad es una alteración específica de las vías piramidales que provoca rigidez, no espasmos.

Pitman, Bliznikas y Baredes (2006) refieren que la DE es un desorden crónico de la voz, caracterizado por una excesiva o inapropiada contracción de los músculos laríngeos durante el habla. Simonyan, Ludlow y Vortmeyer (2010) definen a su vez la DE como una distonía focal laríngea de patofisiología desconocida, caracterizada por espasmos involuntarios en los músculos laríngeos durante la producción de habla. En las distonías laríngeas hay alteraciones del tono muscular que afectan la movilidad de cuerdas vocales. Bliznikas (2004) refiere que la DE es una de las alteraciones más complicadas de tratar, a pesar de que se han hecho variados intentos para mejorar los síntomas de esta enfermedad. El motivo de esta dificultad terapéutica es que la causa está aún indeterminada, por lo que el tratamiento de este desorden continúa dirigido a aliviar el espasmo sintomático vocal, más que a curarlo. Según Pitman et al. (2006) la electromiografía revela en los pacientes con DE anormalidades en el movimiento voluntario de las cuerdas vocales. Estos pacientes presentan niveles anormalmente altos de actividad residual en ambos músculos tiroaritenoideo y cricotiroideo, y una falla en el balance ente ambos, que resulta en un aumento de la aducción glótica y tensión de la dimensión anteroposterior durante el habla, la deglución y la respiración. En la DE puede existir un aumento de sensibilidad de los grupos de motoneuronas en el sistema cerebral. Estos autores citan a Zweig, quien en 1988 expresó que los estudios electrofisiológicos informan sobre disfunción en la vía piramidal y en los ganglios basales. Asimismo agregan que unos pocos estudios bioquímicos de autopsias cerebrales han demostrado alteración en los niveles de los neurotransmisores en varias regiones del cerebro medio.

Simonyan et al. (2010) refieren exámenes del tejido cerebral de dos pacientes post mortem, quienes sufrían de DE, y los compararon con cuatro sujetos de un grupo control. Encontraron pequeñas zonas de inflamación en la formación reticular circundante al tracto solitario, el trigémino espinal y el núcleo ambiguo, en la oliva inferior y en la pirámide. Observaron degeneración neuronal y despigmentación en la pars compacta de la sustancia negra y en el locus coerulus. No encontraron acumulación 
anormal de proteínas ni desmielinización o degeneración axonal. Los autores destacan que estos hallazgos neuropatológicos proveen información sobre la patofisiología de la DE.

Ludlow (2011) opina no obstante que poco se sabe acerca del origen de la DE. Se la clasifica como una distonía focal secundaria a un mecanismo neurobiológico desconocido, que produce una anormalidad crónica de la regulación motora laríngea durante el habla y que se desarrolla primariamente en mujeres. La autora agrega que unos pocos casos con mutaciones en THAP1, un gen implicado en regulación de la transcripción, sugieren que una débil predisposición genética puede contribuir a los procesos que causan una anomalía en el control neuronal motor para el habla pero no para las expresiones vocales emocionales, por eso no interfiere con la respiración, llanto, risa y grito.

Blitzer, Brin y Stewart (1998) informan de que a pesar de que las bases genéticas de la DE no están establecidas, un $12.1 \%$ de los pacientes reportan a familiares con los mismos problemas u otras distonías. Muller (2009) sostiene que se distinguen en la actualidad, por criterio genético, 17 distonías primarias: 12 formas autosómicodominantes, 5 autosómico-recesivas. Este autor describe tres tipos de distonías primarias que comprometen a los músculos de la laringe:

-Distonia early onset dystonia, idiopathic torsion dystonia, dystonia musculorum deformans DYT1. Es la más común durante la niñez. Generalmente los primeros síntomas ocurren en los miembros y la distonía se generaliza pocos años después del comienzo. Puede aparecer durante la adolescencia y en la temprana juventud y en algunos casos no se generaliza, quedando focal o segmental. La localización de la enfermedad en la DYT 1 se ha asignado al brazo prolongado del cromosoma 9 (9q34)2. -Distonía whispering dysphonia DYT4. Se ha descripto en una gran familia australiana de 20 enfermos. En 3 de ellos la disfonía ha sido la primera manifestación y fue descripta como "disfonía con voz de susurro". El inicio de la enfermedad ha variado $\begin{array}{lllll}\text { entre } & 13 & y & 37 & \text { años. }\end{array}$ -Distonía idiopathic torsin dystonia of mixed type, DYT6, THAP1. Va progresando frecuentemente a una distonía generalizada. En el inicio aparece en los músculos cervicales o del cráneo. La disfonía es una alteración frecuente. La edad de inicio se produce durante la niñez y adolescencia, aunque también puede ocurrir en la adultez (rango entre 9 y 49 años). 
Los investigadores han estudiado los signos y síntomas y han elaborado diversas clasificaciones de esta patología. Micheli (2002) distingue dos tipos de DE: en aducción y en abducción. En aducción la característica de voz es tensa, estrangulada, con interrupciones o cortes asociados con hiperaducción de cuerdas vocales. Dichas interrupciones no aparecen o reducen su severidad durante actividades como la risa, aclarar la garganta, toser, murmurar, o sonidos en el registro liviano o falsete. En la disfonía abductora, la voz está caracterizada por cortes intermitentes suspirosos, asociados con una prolongada abducción de las cuerdas vocales durante las consonantes sordas en el habla (Ludlow, Naunton, Terada y Anderson, 1991).

Barkmeier, Case y Ludlow (2001) refieren que además de la típica presentación de los síntomas para aductora y abductora, los pacientes pueden exhibir otros síntomas adicionales: tremor o temblor vocal o disfonía por tensión muscular. A su vez, Edgar, Sapienza, Bidus, y Ludlow, (2001) refieren que los subtipos de DE incluyen aductora, abductora y mixtas y además postulan que el tremor puede existir con ambas.

Sapienza y Walton (1999) han estudiado la DE mediante el análisis de tres alteraciones acústicas: segmentos aperiódicos, cambios de frecuencia y cortes fonatorios, mediante la emisión de vocal sostenida y de una lectura. Las autoras han comparado los resultados obtenidos entre los estudios acústicos de una población de casos y los de un grupo control. Los estudios indicaron que los pacientes con DE produjeron mayor cantidad de eventos acústicos aberrantes que en el grupo control. En la vocal sostenida hubo un gran porcentaje de segmentos aperiódicos seguidos por quiebres fonatorios y cambios frecuenciales. Durante la lectura, los cambios frecuenciales han sido el evento acústico predominante.

Roy, Whitchurch, Merrill, Houtz y Smith (2008) realizaron un estudio sobre el análisis de quiebres fonatorios durante la emisión de una frase. Investigaron a dos grupos de pacientes: con disfonías espasmódicas aductoras y con disfonías por tensión muscular. Determinaron la presencia, frecuencia y duración de los quiebres fonatorios, definidos como una completa interrupción de la fonación. Encontraron que el grupo de pacientes con DE presentó un número más alto de quiebres comparados a las disfonías por tensión muscular. Este estudio ha sido considerado como un posible test objetivo con precisión diagnóstica para distinguir ambas patologías vocales.

En línea con los trabajos ahora referenciados, los objetivos de nuestro estudio son determinar indicadores que permitan elaborar un diagnóstico diferencial fonoaudiológico de los pacientes con Distonía Laríngea. Así mismo estudiar, con el 
modelo propuesto por los autores Sapienza y Walton (1999) y Roy et al. (2008) ciertas alteraciones acústicas de las vocales sostenidas: media o promedio de la frecuencia fundamental, desvío estándar de la frecuencia fundamental, frecuencia fundamental mínima y máxima y su rango o diferencia y el número de quiebres o interrupciones fonatorias, en un grupo de pacientes con distonía espasmódica y comparar los resultados con los obtenidos en un grupo control, a través de valores descriptivos.

La investigación se lleva a cabo siguiendo la hipótesis de que los pacientes portadores de DE presentan alteraciones de la voz, con resultados acústicos diferentes, a las emisiones vocales de otros sujetos sin daños estructurales en la mucosa glótica ni alteraciones neurológicas que involucren la dinámica cordal.

\section{Método}

\section{Materiales}

Se han estudiado parámetros fonético-acústicos de una población de pacientes con DE, quienes han consultado para su diagnóstico y tratamiento, en la División ORL del Hospital de Clínicas “José de San Martín”, en el período comprendido entre el año 2000 y el 2012. Se utilizaron para este trabajo emisiones grabadas con la utilización de un micrófono unidireccional y la placa de audio Sound Blaster, en una sala sin tratamiento acústico. Los parámetros fueron investigados también en las emisiones de un grupo control.

\section{Procedimiento}

Se grabó a los pacientes y a los sujetos del grupo control dos emisiones de la vocal /a/ sostenida con el micrófono colocado a $10 \mathrm{~cm}$ de distancia de la boca para establecer la concordancia de resultados entre ambas, en una frecuencia o tono cómodo, con la consigna "Diga una /a/ prolongada". Se seleccionó para este estudio una de ellas al azar. Se utilizó el Sistema de Análisis Acústico Praat (Boersma y Weenink, 2003) con el cual se investigaron las variables que a continuación se detallan. Estas señales investigadas han tenido duración aproximada de 3 segundos (ver anexo para ejemplo de uno de los estudios efectuados a una paciente con DE, y a una persona sexo femenino del grupo control). 


\section{Participantes}

Participan en el estudio 83 pacientes con DE de sexo femenino de 30 a 87 años (media de 64,82, desvío estándar de 10,36) y 24 pacientes de sexo masculino, de 30 a 82 años de edad (media 60, desvío estándar de 12,16). Se conformó un grupo control de 112 sujetos: 64 personas de sexo femenino de 30 a 88 años (media 60,5, desvío estándar 12) y de 48 de sexo masculino de 30 a 87 años de edad (media 61, desvío estándar 15). Estos sujetos no presentaban daños estructurales en la mucosa glótica ni alteraciones neurológicas que involucraran la dinámica cordal.

Se observa en la Tabla 1 que se ha distribuido a la población según rangos etarios, dadas las modificaciones que se producen en el sistema fonatorio en el proceso de envejecimiento. Asimismo se los ha dividido por sexo por sus diferencias en los valores de Frecuencia Fundamental. Se excluyeron del estudio pacientes con DE, cuyo tratamiento médico quirúrgico ha sido el de infiltración reciente de toxina botulínica. Se aceptaron en la investigación a pacientes con aplicaciones previas de toxina botulínica de más de 12 meses a la fecha de la grabación de las voces. Quedaron fuera del estudio pacientes con tratamiento fonoaudiológico en curso.

Tabla1: Distribución de la población según sexo, rangos etarios y patología- normalidad

\begin{tabular}{|c|c|c|c|c|c|c|c|c|}
\hline & \multicolumn{2}{|c|}{$\begin{array}{l}\text { pacientes } \\
\text { femeninos }\end{array}$} & \multicolumn{2}{|c|}{$\begin{array}{c}\text { pacientes } \\
\text { masculinos }\end{array}$} & \multicolumn{2}{|c|}{$\begin{array}{c}\text { control } \\
\text { femeninos }\end{array}$} & \multicolumn{2}{|c|}{$\begin{array}{c}\text { control } \\
\text { masculinos }\end{array}$} \\
\hline edades & & $\%$ & & $\%$ & & $\%$ & & $\%$ \\
\hline $30-39$ & 1 & 1.20 & 1 & 4.16 & 3 & 4.68 & 4 & 8.33 \\
\hline $40-49$ & 6 & 7.22 & 4 & 16.66 & 9 & 14.06 & 9 & 18.75 \\
\hline $50-59$ & 17 & 20.48 & 7 & 29.16 & 14 & 21.87 & 6 & 12.50 \\
\hline $60-69$ & 28 & 33.73 & 5 & 20.83 & 21 & 32.81 & 11 & 22.91 \\
\hline $70-79$ & 28 & 33.73 & 4 & 16.66 & 14 & 21.87 & 13 & 27.08 \\
\hline $80-89$ & 3 & 3.61 & 3 & 12.50 & 3 & 4.68 & 5 & 10.41 \\
\hline $\mathrm{N}$ & 83 & $100 \%$ & 24 & $100 \%$ & 64 & $100 \%$ & 48 & $100 \%$ \\
\hline Min & 36 & & 39 & & 36 & & 31 & \\
\hline Máx. & 87 & & 82 & & 88 & & 87 & \\
\hline Rango & 51 & & 43 & & 52 & & 56 & \\
\hline media & 64,82 & & 60,00 & & 60,50 & & 61,00 & \\
\hline D. estándar & 10,36 & & 12,16 & & 12,00 & & 15,00 & \\
\hline
\end{tabular}




\section{Resultados}

La primera variable evaluada fue la frecuencia fundamental (F0 en adelante). Se obtuvieron los valores de F0 en las señales vocálicas de cada uno de los sujetos de ambos grupos. Se calculó el promedio en cada uno de ellos.

Como se observa en la tabla 2, se comparan los promedios de valores obtenidos de la F0 en el sexo femenino. En esta población, los valores promedio de F0 presentan cierta variabilidad entre un rango etario y otro. En el grupo de controles los valores de F0 se mantienen prácticamente uniformes para los diferentes rangos etarios, salvo el último grupo de 80 a 89 años, en los que la frecuencia fundamental desciende considerablemente.

Tabla 2: Comparaciones de los promedios de F0 según rangos etarios, en casos y controles femeninos

\begin{tabular}{ccccc}
\hline Femenino & \multicolumn{2}{c}{ Pacientes $(\mathrm{n}: 83)$} & \multicolumn{2}{c}{ Controles $(\mathrm{n}: 64)$} \\
\hline Edades & $\mathrm{n}$ & $\mathrm{F} 0(\mathrm{~Hz})$ & $\mathrm{n}$ & $\mathrm{F} 0(\mathrm{~Hz})$ \\
\hline $30-39$ & 1 & 368.351 & 3 & 202.275 \\
$40-49$ & 6 & 163.526 & 9 & 199.501 \\
$50-59$ & 17 & 183.748 & 14 & 193.904 \\
$60-69$ & 28 & 179.143 & 21 & 195.896 \\
$70-79$ & 28 & 196.974 & 14 & 198.352 \\
$80-89$ & 3 & 174.588 & 3 & 149.432 \\
\hline & Prom. & 211.055 & Prom. & 189.899
\end{tabular}

Aplicando los siguientes test estadísticos, no se han encontrado diferencias significativas en los promedios de F0 entre las poblaciones de pacientes y grupo control de sexo femenino (Welch: $\mathrm{t}=-1.5988, \mathrm{gl}=135.85, \mathrm{p}=0.1122$; Wilcoxon: $\mathrm{W}=2167, \mathrm{p}$ $=0.04395)$.

Como se muestra en la tabla 3, respecto a la F0 en el sexo masculino, se comparan los promedios de valores obtenidos de la frecuencia fundamental, en el grupo de 24 pacientes, de 30 a 82 años con los valores obtenidos en el grupo control de 48 sujetos de 30 a 87 años de edad. En la población de pacientes de sexo masculino, los valores de F0 presentan cierta variabilidad entre un rango etario y otro. En el grupo de controles los valores de F0 no se han mantenido uniformes para los diferentes rangos etarios. El valor es más alto en el rango etario de 30 a 39 años, va descendiendo hacia 
los 50-59 años, para aumentar nuevamente en los rangos 70 a 79 y 80 a 89 años, en los que la frecuencia fundamental está más elevada.

Tabla 3: Comparaciones de los promedios de frecuencia fundamental según rangos etarios, en casos y controles masculinos:

\begin{tabular}{ccccc}
\hline Masculino & \multicolumn{2}{c}{ Pacientes } & & Controles \\
\hline Edades & $\mathrm{n}$ & $\mathrm{F} 0(\mathrm{~Hz})$ & $\mathrm{n}$ & $\mathrm{F} 0(\mathrm{~Hz})$ \\
\hline $30-39$ & 1 & 152.663 & 4 & 141.355 \\
$40-49$ & 4 & 214.865 & 9 & 122.673 \\
$50-59$ & 7 & 137.675 & 6 & 115.088 \\
$60-69$ & 5 & 122.397 & 11 & 131.530 \\
$70-79$ & 4 & 111.358 & 4 & 144.804 \\
$80-89$ & 3 & 145.536 & 5 & 142.181 \\
\hline & Prom. & 147.415 & Prom. & 132.938 \\
\hline
\end{tabular}

Aplicando los test estadísticos no se encontraron diferencias significativas en los promedios de F0 entre la población de pacientes y el grupo control en el sexo masculino: $($ Welch: $\mathrm{t}=1.7011, \mathrm{gl}=27.488, \mathrm{p}=0.1002$; Wilcoxon: $\mathrm{W}=677, \mathrm{p}=$ $0.2317)$

Con respecto al desvío estándar de F0, en el caso del sexo femenino se comparan los promedios entre la población de pacientes y el grupo control. En la figura 1 se observa una gran diferencia entre ambos grupos, según rangos etarios, respecto de los valores obtenidos para el desvío estándar de F0 (ver anexo para los resultados del desvío estándar de F0 en los informes vocales de una paciente y de una persona del grupo F0 control).

En el sexo masculino, los promedios de valores del desvío estándar de la F0 entre la población de pacientes y el grupo control muestran una gran diferencia de los valores obtenidos entre ambos grupos -ver figura 2. 


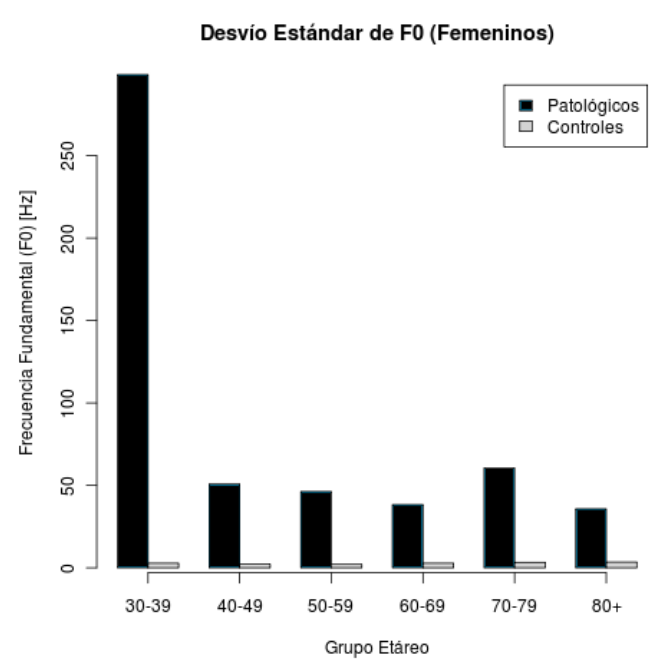

Figura 1: comparación entre valores del desvío estándar de F0 en casos con DE y controles, del sexo femenino

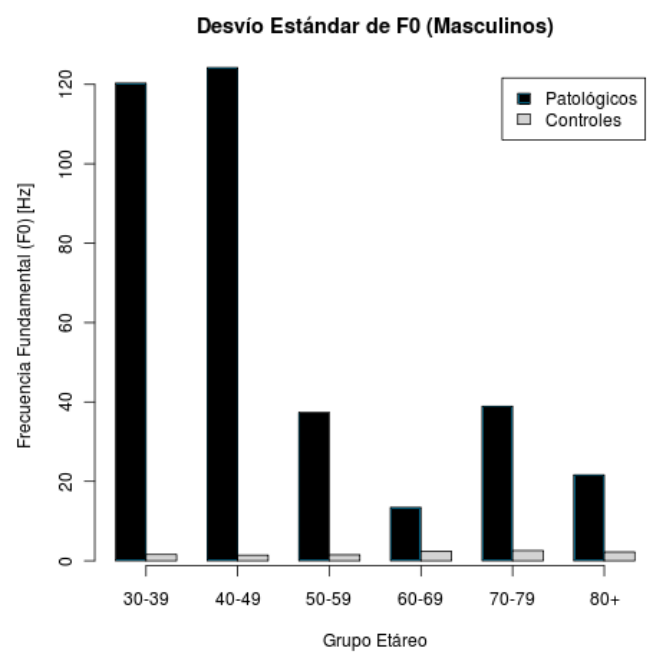

Figura 2: comparación entre los valores del desvío estándar de F0 en casos con DE y controles en sexo masculino

A su vez, en la tabla 4 se observa el valor promedio de los desvíos estándar de la frecuencia fundamental, obtenidos del análisis de la emisión de pacientes y controles de ambos sexos. Asimismo los valores máximo y mínimo obtenidos.

Aplicando los test estadísticos se encontraron diferencias significativas con un 95\% de confianza en las medias de desvío estándar de F0 entre la población de pacientes y grupo control (Welch: $\mathrm{t}=9.1756, \mathrm{gl}=107.14, \mathrm{p}<<0.001$; Wilcoxon: $\mathrm{W}=$ $11855, \mathrm{p}<0.001)$. 
Tabla 4: Comparación de los valores promedio de desvío estándar de F0 y valor máximo y mínimo de la misma variable, separados según sexo.

\begin{tabular}{lrrrr}
\hline & \multicolumn{2}{c}{ Femenino } & \multicolumn{2}{c}{ Masculino } \\
\hline & Pacientes & Controles & Pacientes & Controles \\
\hline Desv estándar prom.(Hz) & 51.218 & 2.707 & 48.610 & 2.055 \\
Valor máximo (Hz) & 299.192 & 8.608 & 263.782 & 3.931 \\
Valor mínimo (Hz) & 2.972 & 950 & 2.020 & 712 \\
\hline
\end{tabular}

Para estudiar la variable Rango o Diferencia en el valor de F0, se ha restado el valor mínimo del máximo de la frecuencia fundamental (Pitch) durante la emisión de la vocal prolongada (ver anexo). En el sexo femenino se comparan los promedios de los rangos entre la frecuencia máxima y mínima de F0, durante la emisión de vocal prolongada, en la población de pacientes y sujetos del grupo control, como puede verse en la figura 3.

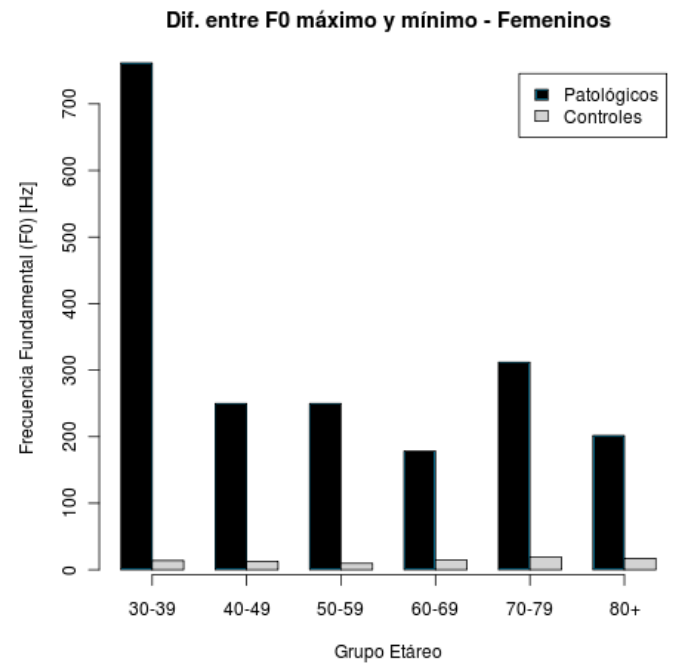

Figura 3: Diferencias entre frecuencia máxima y mínima en casos y controles del sexo femenino, según rango etario.

En el sexo masculino se comparan igualmente los promedios de los rangos entre la frecuencia máxima y mínima de F0, durante la emisión de vocal prolongada, en la población de pacientes y los sujetos del grupo control, como puede verse en la figura 4. 


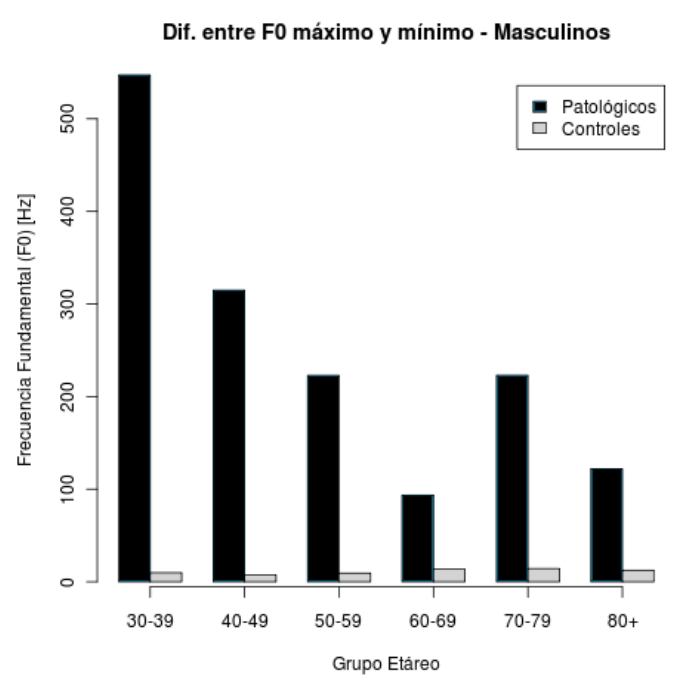

Figura 4: Diferencias entre frecuencia máxima y mínima, en casos y controles del sexo masculino, según rango etario.

En la tabla 5 se observa el valor promedio de las diferencias entre la frecuencia fundamental máxima y mínima, calculado a través de la emisión de vocal prolongada, en pacientes y controles, separados por sexo. Asimismo los valores máximo y mínimo obtenidos.

Tabla 5: Valor promedio de la diferencia entre frecuencia máxima y mínima, a lo largo de /a/ sostenida y valores máximo y mínimo obtenidos.

\begin{tabular}{|c|c|c|c|c|}
\hline & \multicolumn{2}{|c|}{ Femenino } & \multicolumn{2}{|c|}{ Masculino } \\
\hline & Pacientes & Controles & Pacientes & Controles \\
\hline $\begin{array}{l}\text { Diferencia entre la F0 } \\
\text { máxima y mínima }(\mathrm{Hz})\end{array}$ & 250.411 & 14.129 & 212.193 & 11.591 \\
\hline Valor máximo (Hz) & 820.546 & 41.936 & 693.081 & 26.904 \\
\hline Valor mínimo (Hz) & 15.814 & 5.386 & 10.122 & 3.933 \\
\hline
\end{tabular}

Aplicando los cálculos estadísticos se encontraron diferencias significativas con un 95\% de confianza en los valores: promedio de diferencias entre frecuencia máxima y mínima, entre la población con DE y el grupo control. (Welch: $\mathrm{t}=10.2428, \mathrm{gl}=$ 107.209, $\mathrm{p}<0.001$; Wilcoxon: $\mathrm{W}=11863, \mathrm{p}<<0.001)$.

Por último, con respecto a la variable relativa a los quiebres o interrupciones en la señal, se calcularon con el sistema Praat el número de interrupciones en el segmento de 3 segundos aproximados de duración de la vocal /a/ como se muestra en la figura 5. 
No se ha efectuado este cálculo en los grupos control de ambos sexos, ya que no han presentado interrupciones o quiebres a lo largo de las señales emitidas.

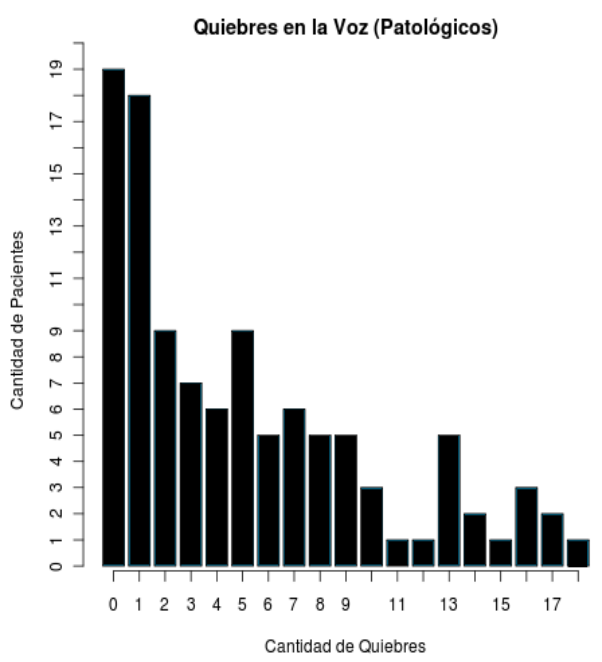

Figura 5: quiebres o interrupciones en la vocal /a/. En abscisas: cantidad de pacientes, en ordenadas cantidad de quiebres.

La tabla 6 muestra, por su parte, el número de interrupciones durante la emisión de la señal en ambos grupos divididos según sexo.

Tabla 6: Registro de las interrupciones en la emisión de /a/ sostenida, de 3 segundos de duración aproximada.

\begin{tabular}{ccc}
\hline & Femenino & Masculino \\
\hline & Pacientes & Pacientes \\
\hline Máximo interrupciones & 18 & 16 \\
Promedio interrupciones & 5 & 5 \\
\hline
\end{tabular}

\section{Discusión}

Como puede verse en la tabla 2, se ha dividido a la población por rangos etarios, dados los cambios que se producen en la estructura de cuerdas vocales durante el proceso de envejecimiento. Se observa que los resultados en la población de controles pueden compararse con los obtenidos por otros investigadores. Así, el valor promedio de F0 obtenido por Elisei (2012) para el sexo femenino en hablantes sin alteración vocal, en la edad cronológica de 20 a 35 años, es de $207 \mathrm{~Hz}$, resultando similar al de $201 \mathrm{~Hz}$. observado por nosotros para el mismo grupo. Sin embargo el valor de F0 obtenido para la población masculina de controles de 30 a 49 años es de: $131 \mathrm{~Hz}(132.014 \mathrm{~Hz})$, levemente elevado respecto a los $119 \mathrm{~Hz}$ de Elisei (2012). 
El valor promedio de F0 en la población femenina de controles, de 60 a 88 años es de $198 \mathrm{~Hz}(197.828 \mathrm{~Hz}$ ), se acerca a $203.0 \mathrm{~Hz}$ obtenido por Méndez et al. (2010), en una población femenina de 60 a 85 años. El valor promedio de F0 en la población masculina de controles en el grupo de 60 a 87 años es de $139 \mathrm{~Hz}(139.505 \mathrm{~Hz}$ ), presentando cierta diferencia de los $159.38 \mathrm{~Hz}$ obtenidos por Méndez et al. (2010), en una población masculina de 60 a 85 años.

Es interesante destacar la variabilidad de resultados de la F0 en la población de pacientes. En la tabla 3 se observa que en la población femenina con DE, el valor de F0 máximo obtenido ha sido $368 \mathrm{~Hz}$., perteneciente a una paciente en el rango etario $30 \mathrm{a}$ 39 años de edad, y el mínimo 113 Hz., en el rango etario de 60 a 69 años.

En la población masculina con DE los valores máximos y mínimos de F0 obtenidos han sido $324 \mathrm{~Hz}$. y $77 \mathrm{~Hz}$. respectivamente. De estos resultados se puede inferir la dificultad que los pacientes con distonías presentan en el control motor muscular de la fonación, ya que es probable que coloquen en posición alta la laringe, como medio de compensación fonatoria, con el resultante de F0 elevada, o por el contrario emiten en registro frito, cuya característica es el valor muy descendido de frecuencia fundamental.

Se destaca que respecto a la primera variable en estudio, la que se refería al valor de la F0, la aplicación de los tests estadísticos no ha dado diferencias significativas entre los grupos de pacientes y control en ambos sexos, con lo cual el valor de esta variable no puede ser tomada como indicador de la presencia de DE, según la hipótesis que da lugar a esta investigación.

Con respecto a la segunda variable, la relativa al desvío estándar, como se observa en las figuras 1 y 2, la comparación de valores del desvío estándar de F0 en DE, respecto a controles en ambos sexos, muestra que existe una notable diferencia entre las emisiones de los sujetos con DE y el grupo control, por el alto grado de dispersión o variabilidad del valor de la F0, característica que también se comprobará en el análisis de los resultados de la tercera variable, que es la diferencia entre la frecuencia máxima y mínima durante la emisión de la señal.

En la tabla 4 se observan los promedios de desvíos estándar de F0. En el grupo de pacientes femeninas el promedio de los desvíos estándar es de $51.218 \mathrm{~Hz}$. y el promedio de los desvíos estándar del grupo control es de $2.707 \mathrm{~Hz}$. En el grupo de pacientes masculinos el promedio de los desvíos estándar de F0 es de $48.610 \mathrm{~Hz}$. y en 
el grupo control es de $2.055 \mathrm{~Hz}$. Con la aplicación de test estadísticos se muestran diferencias significativas entre ambas poblaciones.

La tercera variable de estudio, el rango o diferencia en el valor de la F0 (cambios frecuenciales) durante la emisión de vocal prolongada ha evidenciado en los estudios acústicos que los pacientes investigados no pueden sostener una F0 estable durante la emisión prolongada de la vocal /a/. Esta patología, la distonía laríngea, produce habitualmente una gran inestabilidad en el funcionamiento muscular laríngeo y en la oscilación de las cuerdas vocales, con modificaciones bruscas en su dinámica durante la fonación, lo que puede provocar cambios frecuenciales. Edgar, Sapienza y Ludlow, (2001) han definido al cambio frecuencial como una diferencia de $50 \mathrm{~Hz}$. o más en la F0, dentro de los 50 milisegundos. Esta variable se grafica en las figuras 3 y 4 , y en la tabla 5, que muestran la notable diferencia en el rango entre frecuencia máxima y mínima, entre ambas poblaciones de casos y controles, separadas por rangos etarios, para sexo femenino y masculino respectivamente, diferencias más marcadas en los primeros rangos etarios de 30 a 39 años para ambos sexos y en 40 a 49 años en los hombres. El promedio de los rangos diferenciales.

Comparando ambas poblaciones, se observa que los pacientes con DE producen emisiones de vocal con un rango diferencial entre frecuencia máxima y mínima muy alto respecto a los sujetos del grupo control. La aplicación de test estadísticos ha dado diferencias significativas entre ambos grupos. Tanto en la población patológica femenina como masculina los valores obtenidos indican que existen fallas en el sostén de una frecuencia estable a lo largo de la emisión, con la presencia de importantes cambios o saltos frecuenciales, y una notable diferencia entre el valor de frecuencia máxima y mínima durante la emisión de vocal sostenida.

Se observa en la figura 5 y en la tabla 6 que en una población de 83 personas de sexo femenino, durante las emisiones de /a/ de 3 segundos de duración aproximada, el número de interrupciones osciló entre 1 y 18, con un promedio de 5. En una población de 24 personas de sexo masculino, durante las emisiones de /a/ de 3 segundos de duración aproximada, el número de interrupciones osciló entre 1 y 16, con un promedio de 5. Existen quiebres o interrupciones en 89 de los 108 pacientes estudiados -recordamos que en el grupo control no se observó ninguno de estos quiebres.

En resumen, los pacientes con distonías laríngeas cuyo correlato vocal es la DE, presentan emisiones con quiebres o interrupciones cuya existencia ya ha sido referida 
por los autores en el tema. Los sujetos del grupo control investigados, no presentaron quiebres o interrupciones en la emisión de la vocal /a/.

\section{Conclusiones}

Se ha trabajado en el objetivo de analizar las características clínico vocales de un grupo de pacientes con DE, sus alteraciones acústicas y compararlas con un grupo control. Respecto a la población estudiada, se observa la prevalencia de mujeres en el grupo de pacientes con DE (coincidiendo en estos resultados con Schweinfurth, Billante y Courey 2002; y Pitman, Bliznikas y Baredes, 2006). En dicho grupo, la mayor cantidad de pacientes está en el rango etario de 50 a 80 años. La población de hombres con DE es mucho menor. Respecto a la variable F0, con la aplicación de tests estadísticos, no se encontraron diferencias significativas para las medias del promedio de F0 para hombres y mujeres. El rango o diferencia entre frecuencia mínima y máxima y valor de desvío estándar son muy elevados en la población de pacientes con DE respecto al grupo control. Se encontraron diferencias significativas en las variables: medias de Desvío Estándar de F0 y en las medias de las diferencias de máximo y mínimo de F0. Existen hasta 18 quiebres o interrupciones durante la emisión de 3 segundos en los pacientes investigados.

Como conclusiones finales observamos que el desvío estándar de F0, el rango entre el valor máximo y mínimo de F0 y el número de interrupciones o quiebres durante una emisión de vocal prolongada, pueden utilizarse como indicadores de la presencia de DE.

\section{Referencias}

Barkmeier, J., Case, J., y Ludlow, C. (2001). Identification of symptoms for spasmodic dysphonia and vocal tremor: A comparison of expert and nonexpert judges. Journal of Communication Disorders, 34, 21-37.

Behlau, M., y Pontes, P. (2002). Las llamadas disfonías espasmódicas. Dificultades de diagnóstico y tratamiento. En J. Menaldi (Ed.), La voz patológica. (pp. 173-203). Buenos Aires: Editorial Médica Panamericana.

Blitzer, A., Brin, M., y Stewart, C (1998). Botulinum toxin management of spasmodic dysphonia: A 12-year experience in more than 900 patients. The Laryngoscope, $108,1435-1441$. 
Boersma, P., y Weenik, D. (2013). PRAAT: Doing phonetics by computer. Versión 5.3.50. Acceso 21 de mayo de 2013 de http:www.praat.org.

Edgar, J., Sapienza, C., Bidus, K., y Ludlow, C. (2001). Acoustic measures of symptoms in abductor spasmodic dysphonia, Journal of Voice, 15, 362-372.

Elisei, N.G. (2012). Análisis acústico de la voz normal y patológica utilizando dos sistemas diferentes: Anagraf y Praat. Revista Interdisciplinaria, 29, 339-357.

Ludlow, C. L., Naunton. R. F., Terada, S., y Anderson, B.J. (1991). Successful treatment of selected cases of abductor spasmodic dysphonia using botulinum toxin injection. Otolaryngology - Head and Neck Surgery, 104, 849-55.

Ludlow, C. (2011) Spasmodic dysphonia: A laryngeal control disorder specific to speech. The Journal of Neuroscience, 31, 793-797.

Lundy, D., Roy, S., Xue, J., Casiano, R., y Jassir, D. (2004). Spastic/spasmodic vs. tremulous vocal quality: Motor speech profile analysis. Journal of Voice, $18,146-152$.

Méndez, A.M., Álvarez Tenorio, M., Bejarano, M.A., y Pulido, C. (2010). Parámetros acústicos de la voz en el adulto mayor. Umbral Científico, 17, 9-17.

Micheli, F. (2002). Distonías. En Micheli, F., Tolosa, E., y Muñoz, E., (Eds.), Tratado de neurología clínica. (pp. 565-582). Buenos Aires: Editorial Médica Panamericana.

Morrison, M., Ramage, L., Nichol, H., Pullan, B., May, P., y Salkeld, L. (1996). Tratamiento de los trastornos de la voz. Barceona: Masson.

Pitman, M., Bliznikas, D., y Baredes, S. (2006). Spasmodic dysphonia, http://eMedicine.com

Roy, N., Whitchurch, M., Merrill, R., Houtz, D., y Smith, M. (2008). Differential diagnosis of adductor spasmodic dysphonia and muscle tension dysphonia using phonatory break analysis, The Laryngoscope, 118, 2245-2253.

Sapienza. C. M., Walton, S., y Thomas, M. (1999). Acoustic variations in adductor spasmodic dysphonia as a function of speech task. Journal of Speech, Language and Hearing Research, 42, 127-140.

Sapienza, C.M., Murry,T., y Walton, S. (2000). Adductor spasmodic dysphonia and muscular tension dysphonia: acoustic analysis of sustained phonation and reading, Journal of Voice, 14, 502-520. 
Schweinfurth, J. M., Billante, M., y Courey, M. S. (2002). Risk factors and demographics in patients with spasmodic dysphonia. The Laryngoscope, 112, 220-223.

Simonyan, K., Ludlow, C., y Vortmeyer, A. (2010). Brainstem pathology in spasmodic dysphonia, The Laryngoscope, 120, 121-124.

Muller, U. (2009). Monogenic primary dystonias. Brain,132, 2005-2025. 


\section{Anexo:}

\section{a) Estudio acústico de la vocal /a/ sostenida:}

Paciente de sexo femenino de 61 años de edad, con diagnóstico otorrinolaringológico de Disfonía espasmódica

Sistema de análisis acústico Praat:

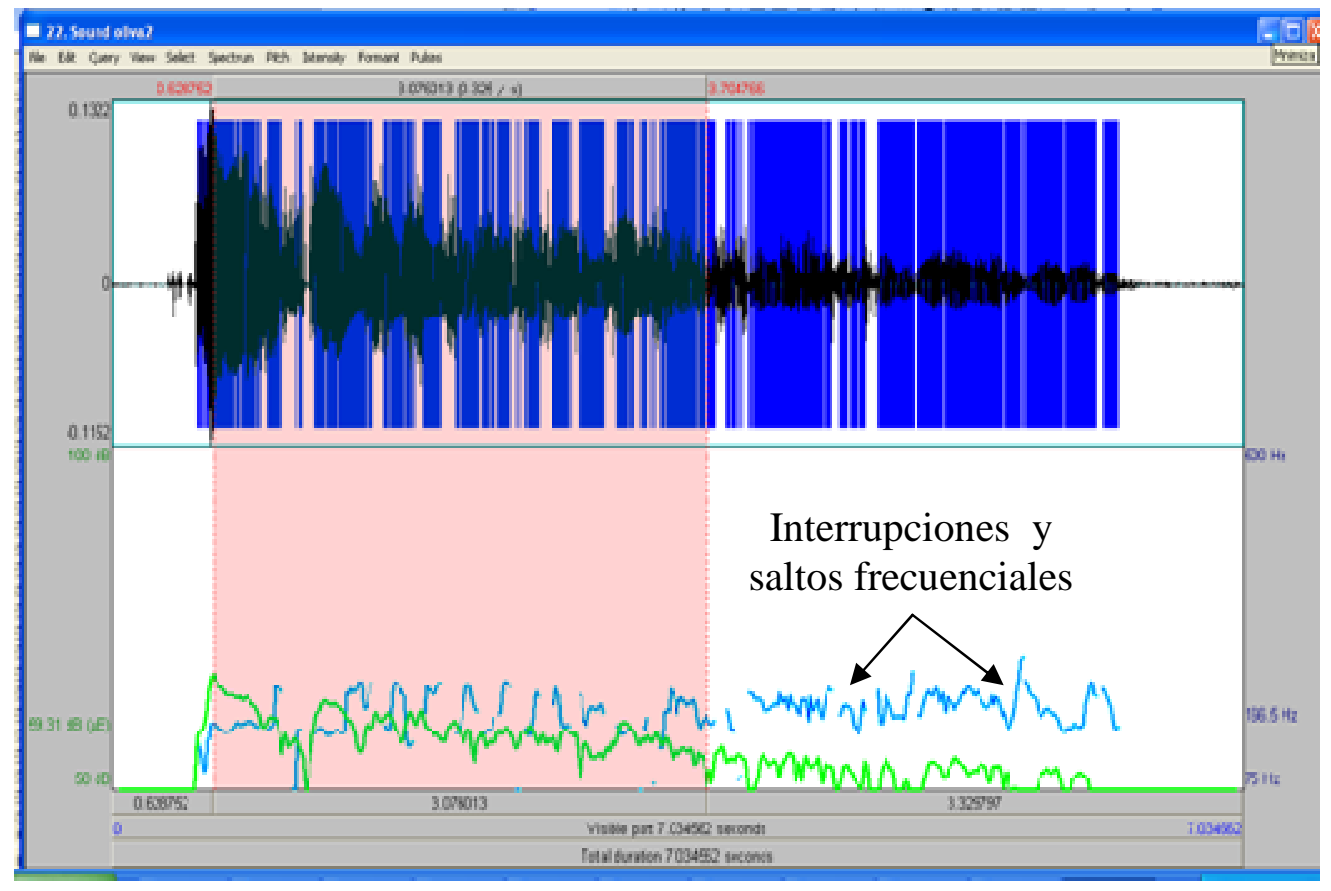

Voice report: (Se han efectuado los cálculos con una ventana de duración de la señal /a/ de 3.076 segundos)

\section{Pitch:}

Median pitch: $161.184 \mathrm{~Hz}$

Mean pitch: $166.512 \mathrm{~Hz}$

Standard deviation: $26.632 \mathrm{~Hz}$

Minimum pitch: $66.348 \mathrm{~Hz}$

Maximum pitch: $211.691 \mathrm{~Hz}$

Rango de variación entre frecuencia mínima y máxima: 145.343 Hz.

\section{Voicing:}

Fraction of locally unvoiced frames: $16.558 \% \quad$ (51/308)

Number of voice breaks: 16

Resultados de este estudio: El valor de Frecuencia fundamental: $166.512 \mathrm{~Hz}(167 \mathrm{~Hz})$, el rango de variación de la Frecuencia fundamental es de $145.343 \mathrm{H}$, (145 Hz), la desviación estándar $26.632 \mathrm{~Hz}(27 \mathrm{~Hz})$, con 16 quiebres o interrupciones fonatorias. 


\section{b) Estudio acústico de la vocal /a/ sostenida, del grupo control:}

Paciente de sexo femenino de 72 años de edad, con diagnóstico de cuerdas vocales sin alteración

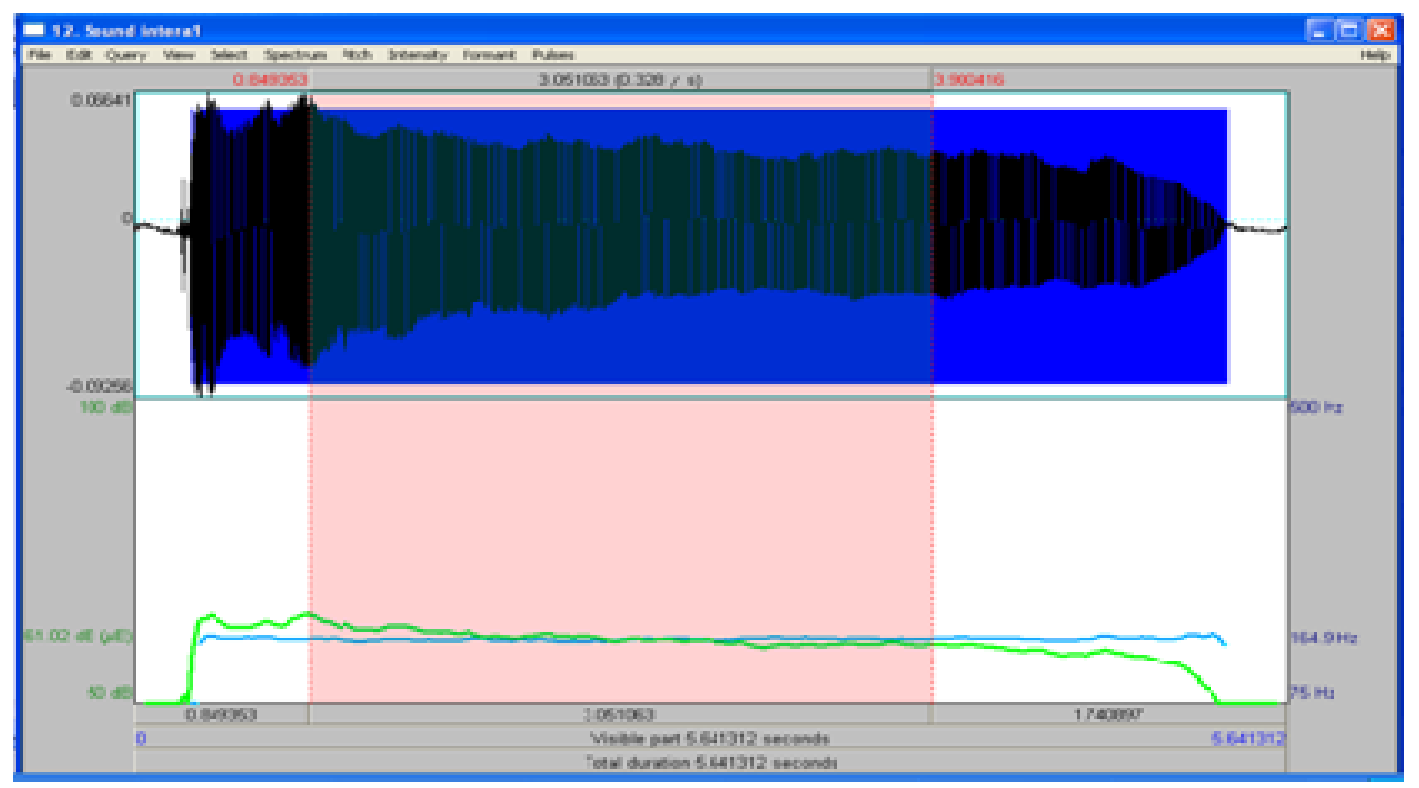

(Se han efectuado los cálculos con una ventana de duración de la señal /a/ de 3.051 segundos)

\section{Pitch:}

Median pitch: $192.305 \mathrm{~Hz}$

Mean pitch: $192.592 \mathrm{~Hz}$

Standard deviation: $1.279 \mathrm{~Hz}$

Minimum pitch: $190.380 \mathrm{~Hz}$

Maximum pitch: $196.709 \mathrm{~Hz}$

Rango de variación entre frecuencia mínima y máxima: $6.329 \mathrm{~Hz}$

\section{Voicing:}

Fraction of locally unvoiced frames: 0 (0/308)

Number of voice breaks: 0

Resultados de este estudio: el valor de Frecuencia Fundamental: $192.592 \mathrm{~Hz}$ (193 Hz), el rango de variación de la Frecuencia fundamental es de $6.329 \mathrm{~Hz}$ (6 Hz), la desviación estándar: $1.279 \mathrm{~Hz}(1 \mathrm{~Hz})$, sin quiebres o interrupciones fonatorias. 